\title{
BMJ Open Changes in vulnerability among older patients with cardiovascular disease in the first 90 days after hospital discharge: A secondary analysis of a cohort study
}

\author{
Jinjiao Wang, ${ }^{1}$ Mary S Dietrich,, ${ }^{2,3,4}$ Susan P Bell, ${ }^{5,6}$ Cathy A Maxwell, ${ }^{2}$ \\ Sandra F Simmons, ${ }^{5,6,7}$ Sunil Kripalani, ${ }^{5,8}$ for the Vanderbilt Inpatient Cohort Study \\ (VICS)
}

To cite: Wang J, Dietrich MS, Bell SP, et al. Changes in vulnerability among older patients with cardiovascular disease in the first 90 days after hospital discharge: A secondary analysis of a cohort study. BMJ Open 2019;9:e024766. doi:10.1136/ bmjopen-2018-024766

- Prepublication history for this paper is available online. To view these files, please visit the journal online (http://dx.doi org/10.1136/bmjopen-2018024766).

Received 14 June 2018 Revised 10 October 2018 Accepted 23 November 2018

D) Check for updates

(c) Author(s) (or their employer(s)) 2019. Re-use permitted under CC BY-NC. No commercial re-use. See rights and permissions. Published by BMJ.

For numbered affiliations see end of article.

\section{Correspondence to} Dr Jinjiao Wang; jinjiao_wang@urmc.rochester. edu

\section{ABSTRACT}

Objectives (1) To compare changes in vulnerability after hospital discharge among older patients with cardiovascular disease who were discharged home with self-care versus a home healthcare $(\mathrm{HHC})$ referral and (2) to examine factors associated with changes in vulnerability in this period.

Design Secondary analysis of longitudinal data from a cohort study.

Participants and setting 834 older ( $\geq 65$ years) patients hospitalised for acute coronary syndromes and/or acute decompensated heart failure who were discharged home with self-care $(n=713)$ or an HHC referral $(n=121)$. Outcome Vulnerability was measured using Vulnerable Elders Survey 13 (VES-13) at baseline (prior to hospital admission) and 30 days and/or 90 days after hospital discharge. Effects of $\mathrm{HHC}$ referral on postdischarge change in vulnerability were examined using three linear regression approaches, with potential confounding on $\mathrm{HHC}$ referral adjusted by propensity score matching.

Results Overall, $44.4 \%$ of the participants were vulnerable at prehospitalisation baseline and $34.4 \%$ were vulnerable at 90 days after hospital discharge. Compared with self-care patients, HHC-referred patients were more vulnerable at baseline (66.9\% vs $40.3 \%)$, had more increase (worsening) in VES-13 score change $(B=-1.34(-2.07,-0.61), p<0.001)$ in the initial 30 days and more decrease (improvement) in VES-13 score change $(B=0.83(0.20,1.45), p=0.01)$ from 30 to 90 days after hospital discharge. Baseline vulnerability and the $\mathrm{HHC}$ referral attributed to $14 \%-16 \%$ of the variance in vulnerability change during the 90 postdischarge days, and $6 \%$ was attributed by patient age, race (AfricanAmerican), depressive symptoms, and outpatient visits and hospitalisations in the past year.

Conclusion After adjusting for preceding vulnerability and covariates, older hospitalised patients with cardiovascular disease referred to $\mathrm{HHC}$ had delayed recovery in vulnerability in first initial 30 days after hospital discharge and greater improvement in vulnerability from 30 to 90 days after hospital discharge. HHC seemed to facilitate improvement in vulnerability among older patients with cardiovascular disease from 30 to 90 days after hospital discharge.
Strengths and limitations of this study

- This was the first study comparing prospective changes in postdischarge vulnerability for up to 90 days after hospital discharge among 834 older patients with cardiovascular disease in different postacute care options (self-care versus having a home healthcare $(\mathrm{HHC})$ referral).

- Follow-up rates were high, that is, $97 \%$ at 30 days after discharge and $94 \%$ at 90 days after hospital discharge.

- Potential confounders on $\mathrm{HHC}$ referral related to patient sociodemographic and clinical variables were controlled for using propensity score matching.

- The measurement of vulnerability (Vulnerable Elders Survey 13 (VES-13)) includes self-perceived health status and physical function but does not include an objective measure of vulnerability (eg, gait speed). Because of the self-report nature of VES-13, subjects not able to communicate clearly were excluded.

- The specific content and amount of HHC received in the study period were not measured.

\section{INTRODUCTION}

Adults aged 65 years or older comprise $14 \%$ of the US population, ${ }^{1}$ yet they account for over $40 \%$ of hospitalisations in the $\mathrm{USA}^{2}$ Cardiovascular disease is the leading cause of hospitalisation in the elderly ${ }^{3}$ and is associated with complex self-care needs ${ }^{4}$ and postdischarge adverse outcomes. ${ }^{56}$ Vulnerability, defined as a lack of functional reserve to stressors that represents a higher risk for health deterioration, ${ }^{7}$ is prevalent $(54 \%)$ among older adults with cardiovascular disease. ${ }^{8}$ Vulnerability increases the risk of disability, ${ }^{9}$ emergency department (ED) visits, ${ }^{8}$ hospital complications and death. ${ }^{10}$ Vulnerability is also dynamic and its level or severity can change in relation to time ${ }^{11}$ and stressors, such as acute illness and hospitalisation. ${ }^{12} \mathrm{~A}$ vicious cycle is possible, where a higher degree of baseline 
vulnerability increases the risk for hospitalisation, ${ }^{13}$ giving rise to further worsening of vulnerability during and after hospital discharge. ${ }^{8}$ To date, few studies have quantified changes in vulnerability after hospital discharge and postacute services that may modify this trajectory for older patients with cardiovascular disease.

In the USA, half of older hospitalised patients are discharged to postacute care that aims to facilitate functional recovery and prevent adverse outcomes. ${ }^{14}$ In 2013, for example, the postacute care sector in the USA incurred US $\$ 59.4$ billion of medical expenditure. ${ }^{15}$ Common US postacute care modalities include: (1) facility-based skilled nursing and physical rehabilitative services for patients who have a substantial need of intensive physical rehabilitation, (2) nursing homes for patients who reside in long-term care facilities prior to the index hospitalisation and (3) home-based Medicare home healthcare (HHC) services for older patients who do not need intensive physical rehabilitation yet are not able to recover independently (ie, with self-care only).$^{1416}$ In particular, HHC is the fastest growing postacute care modality in the USA that provides multidisciplinary services to over one-third of the non-institutionalised older patients. ${ }^{15}$ These HHC services include skilled nursing, physical therapy, occupational therapy, social work and home health aide assistance. ${ }^{17}$ Studies have shown that HHC promotes functional improvement, ${ }^{18}$ reduces the risk of rehospitalisation ${ }^{19}$ and delays nursing home placements. ${ }^{20}$ Medical expenditures for HHC users were also lower with an adjusted cost saving of US $\$ 6433$ in the 365 days after hospital discharge. ${ }^{21}$ As such, it seems that HHC provides efficient and cost-effective services to prevent postdischarge adverse outcomes.

However, evidence has also shown that patients do not benefit equally from postacute care such as HHC, due to the variance in modifiable risk factors for adverse outcomes, such as hospital readmission. ${ }^{22}$ It is thus important that enough HHC be provided to those at the highest risk for adverse outcomes who also have the greatest potential of functional improvement following HHC. One of these modifiable risk factors is vulnerability, which is found in over half of $(54 \%-89.5 \%))$ of older hospitalised patients. ${ }^{812}$ To date, no studies have examined how HHC affects postdischarge changes in vulnerability to functional decline among older adults.

To fill this gap in knowledge, we conducted this study with the following objectives: (1) to compare the changes in vulnerability from baseline (ie, prior to the event triggering the hospitalisation) up to 90 days after hospital discharge in older patients with cardiovascular diseases who were discharged home with or without a referral to postacute $\mathrm{HHC}$ and (2) to examine factors associated with changes in vulnerability between each assessment point (ie, prior to hospital admission (baseline), 30 and 90 days postdischarge). We hypothesised that HHC-referred patients would had greater improvement in vulnerability during the 90-day period following hospital discharge relative to non-HHC referred patients.
METHODS

\section{Overall design and study population}

This study was a secondary analysis of prospective data (October 2011 to December 2015) from a large prospective study about older patients hospitalised for cardiovascular diseases. $^{23}$

\section{Participants and setting}

Participants in the original study were patients admitted to a major university-affiliated hospital for acute coronary syndromes (ACS) and/or acute decompensated heart failure (ADHF). Exclusion criteria were: (1) age $<18$ years; (2) inability to communicate in English; (3) inability to participate due to blindness, hearing difficulties, sedation, significant cognitive impairment of dementia, active mania or psychosis or (4) receiving hospice or end-of-life care. Participants were interviewed in person prior to hospital discharge and followed up over the telephone at 30 and 90 days after hospital discharge. This study was approved by the University Institutional Review Board. A detailed description of all study measures collected in the original study is available elsewhere. ${ }^{23}$

Subjects in this study reflect a subset of participants in the original study who were $\geq 65$ years old, discharged home from the index hospitalisation and had vulnerability assessments at both baseline and 30 or 90 days after hospital discharge $(\mathrm{n}=834)$. The flow diagram of eligibility screening, enrolment and sample selection is shown in figure 1. Overall, 97\% (807/834) and 94\% (784/834) of the participants in this study completed follow-up assessments, respectively, at 30 days and 90 days after hospital discharge.

\section{Patient and public involvement}

In this study, we used deidentified data from the original cohort study with no direct involvement of or interaction with participants in the design, recruitment or conduct of this study.

\section{Variables and measures}

The dependent variable was vulnerability, as measured by the Vulnerable Elders Survey 13 (VES-13). The VES-13 is a validated self-report measurement, including items on age, self-reported health, ability to complete common physical tasks and difficulties with (independent) activities of daily living (ADLs). According to total VES-13 score, vulnerability was categorised into three categories: being not vulnerable (0-2), vulnerable (3-6) and extremely vulnerable $(7-10) .{ }^{7}$ The VES-13 has strong predictive validity (receiver operating characteristic curve 0.78 ) for long-term functional decline and mortality. ${ }^{791324}$ When assessing baseline vulnerability, patients were asked to recall functional status prior to hospital admission.

The independent variable was the HHC referral, which was determined at hospital discharge by hospital personnel for patients who are homebound and in need of skilled nursing/therapy services, as verified by a physician. ${ }^{25}$ Willingness to accept the HHC referral was confirmed with the patient. 


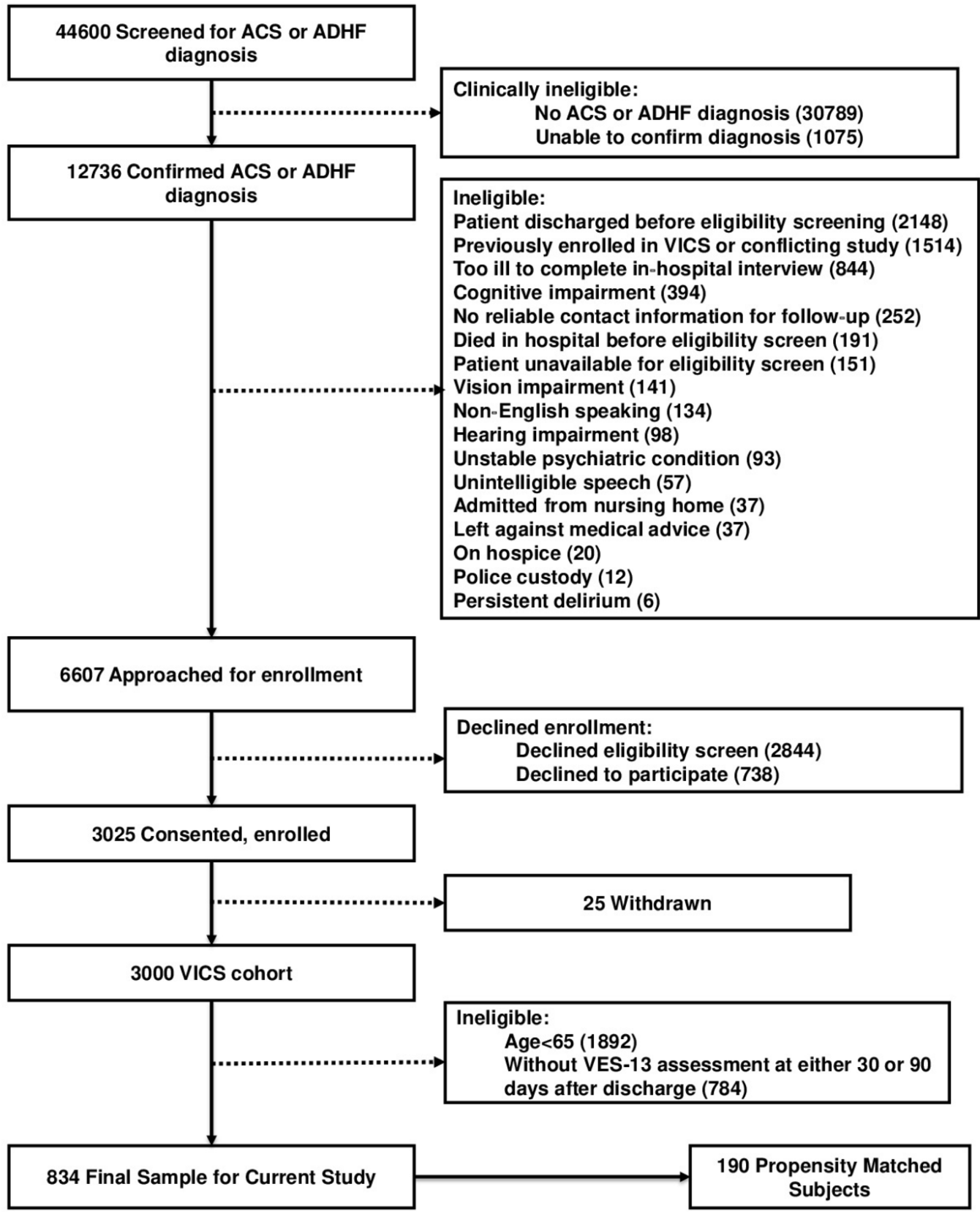

Figure 1 Study flow diagram. ACS, acute coronary syndrome; ADHF, acute decompensated heart failure; VES-13, Vulnerable Elders Survey 13; VICS, Vanderbilt Inpatient Cohort Study.

Covariates for risk adjustment included: (1) demographic and socioeconomic variables: age, sex, race/ ethnicity, education level, health literacy (3-item Brief Health Literacy Screen), ${ }^{26}$ annual household income, difficulty paying bills, marital status, social support (ENRICHD Social Support Inventory) ${ }^{27}$ and (2) health history variables: diagnosis of the index hospitalisation (ACS and/or AHDF), comorbidity (Elixhauser Index), ${ }^{28}$ length of hospital stay, depressive symptoms (Patient Health Questionnaire-8), ${ }^{29}$ cognitive functioning (Short Portable Mental Status Questionnaire) ${ }^{30}$ and previous utilisation of health services (number of outpatient visits, $\mathrm{ED}$ visits and hospitalisations in the past 12 months (at any institution)). These variables were collected at hospital admission from electronic medical record data and face-to-face interviews conducted by trained research personnel using standardised questions and validated measures. Selection of the covariates was based on a conceptual framework on characteristics related to postdischarge patient outcomes developed as part of the original study (citation blinded). ${ }^{23}$

\section{Statistical analysis}

Descriptive statistics were used to evaluate the distribution of study variables for outliers, sparsity of categories and other distributional characteristics. Frequency distributions were used to summarise categorical variables. Due to skewness, continuous variables were 
summarised using the median and IQR and were transformed to normal distributions or into meaningful ordinal categories (dummy coded) for inclusion in analyses with underlying parametric assumptions. $\chi^{2}$ tests of independence and Mann-Whitney tests were used to compare patient variables for HHC-referred and non-HHC-referred (ie, self-care) groups. No missing data were found in the covariates. Missing data in VES-13 scores were found at 30 days (missing $n=27$, total $\mathrm{n}=807$ ) and 90 days (missing $\mathrm{n}=50$, total $\mathrm{n}=784$ ) after discharge and were addressed using listwise deletion. Patients with VES-13 score at baseline and at least one follow-up time point (30 days and/or 90 days) were included in inferential analysis.

Three linear regression approaches were used to examine the effects of HHC referral on change in postdischarge VES-13 scores from baseline: (1) full model: HHC referral indicator and all covariates (full sample); (2) propensity model: HHC referral indicator and propensity score in lieu of the individual covariates (full sample) and (3) propensity-matched subsample: HHC referral indicator only using a subsample of propensity-matched patient pairs. The propensity of HHC referral was calculated from the set of demographic, socioeconomic and health history covariates, that is, the same covariates included in the full model (18 baseline variables). Each HHC patient was manually matched to a non-HHC patient with the closest propensity score (maximum calliper/ difference $=0.012$ ). This process resulted in a subsample of 95 matched cases (total $\mathrm{n}=190$ ) for the matched pair analysis. The dependent variable for each regression model was change in postdischarge VES-13 scores during the respective time period (baseline to 30 days postdischarge, 30 to 90 days postdischarge and baseline to 90 days postdischarge). Because a higher VES-13 score indicates greater vulnerability, a positive change value suggests increasing vulnerability. To control for the effects of initial vulnerability level on 'opportunity for change', baseline VES-13 score was included with HHC referral in the initial step, except for the analysis of change from 30 to 90 days postdischarge, where VES-13 score at 30 days postdischarge was included with HHC referral. All other variables included in each of the regression models were baseline characteristics and measure scores or hospital discharge characteristics (eg, HHC referral). No multiple assessments of within-subject effects were included in these analyses. Effect sizes for HHC referral were generated from each model and evaluated for replication of findings. Finally, hierarchical linear regression models were used to estimate the effects of the set of covariates on the amount of change in vulnerability during the three assessment periods. The (adjusted) $\mathrm{R}^{2}$ change in each model after accounting for the initial period VES-13 score and HHC referral was used for these estimates. An alpha of 0.05 was used for determining statistical significance throughout this study. When pairwise post hoc tests were necessary, a Bonferroni-corrected alpha value was used.
RESULTS

\section{Sample characteristics}

The overall sample included 834 participants who were primarily Caucasian $(90 \%)$ with a median age of 71 years. Of the participants, $40 \%$ were female, $32 \%$ were unmarried, $40 \%$ had an educational level of less than a high school graduation, $18 \%$ had inadequate health literacy and $32 \%$ reported difficulty paying monthly bills. In terms of health history, $35 \%$ were admitted with ADHF, $9 \%$ had mild to moderate cognitive impairment and $28 \%$ had moderate to severe depressive symptoms. The median length of stay of the index hospitalisation was 3 days (range: 1-25 days). Statistically significant differences existed between participants who were referred to HHC on hospital discharge $(n=121)$ and those who were not referred to HHC $(n=713)$ (table 1). None of these between-group differences remained for the propensity-matched pairs $(n=190)$.

\section{Changes in vulnerability: HHC-referred versus non-HHC- referred patients}

Overall, 97\% (807/834) and 94\% (784/834) of the participants in this study completed follow-up assessments, respectively, at 30 days and 90 days after hospital discharge. Reasons of missing follow-up assessments include loss to follow-up, refused interview, withdrawal and death.

Among all study participants $(n=843)$, the rate of vulnerability (VES-13 score $\geq 3$ ) was $44.1 \%$ at baseline, which decreased (ie, improved) to $39.2 \%$ at 30 days and $34.4 \%$ at 90 days postdischarge (table 2). At baseline, $66.9 \%$ of the HHC-referred patients and $40.3 \%$ of the non-HHC-referred patients were vulnerable. In the HHC-referred group, the rate of vulnerability increased to $68.7 \%$ in the initial 30 days after discharge, then decreased to $56.7 \%$ at 90 days postdischarge. In the non-HHC-referred group, the rate of vulnerability continued to decrease over the entire 90-day postdischarge period ( $40.3 \%$ at baseline to $34.3 \%$ after 30 days and $30.8 \%$ after 90 days; table 2 ).

As shown in table 3, the effects of HHC referral on change in postdischarge vulnerability were well replicated among the three regression models using both the entire sample and the propensity-matched pairs. From baseline to 30 days postdischarge, while consistent with the covariate models $(\mathrm{p}<0.001)$, the effects observed in the propensity matched subsample were the strongest (change in VES-13 score $=-1.34(95 \% \mathrm{CI}=-2.07$ to $-0.61), \mathrm{p}<0.001)$. In other words, compared with patients not referred to HHC, the HHC-referred patients had a 0.6-2.1 point increase in VES-13 score (total 10 points) from baseline to 30 days postdischarge. Between 30 and 90 days postdischarge, the differences between the groups in their respective patterns of change reversed, with patients referred to HHC demonstrating a greater decrease in vulnerability than those not-referred to HHC (propensity-matched model, change in VES-13 score $=0.83$ $(95 \% \mathrm{CI}=0.20$ to 1.45$), \mathrm{p}=0.010)$. Figure 2 illustrates these 
Table 1 Characteristics of the sample $(\mathrm{N}=834)$ by HHC referral groups

\begin{tabular}{|c|c|c|c|c|}
\hline Characteristics & $\begin{array}{l}\text { Overall sample } \\
\text { (Total } \mathrm{N}=834 \text { ) }\end{array}$ & $\begin{array}{l}\text { Non-HHC referred } \\
(n=713)\end{array}$ & $\begin{array}{l}\text { HHC referred } \\
(n=121)\end{array}$ & P value \\
\hline \multicolumn{5}{|l|}{ Demographic and socioeconomic status } \\
\hline Female, \% (n) & $40.5(338)$ & $39.1(279)$ & $48.8(59)$ & 0.046 \\
\hline Caucasian/white, \% (n) & $90.8(757)$ & $91.4(652)$ & $86.8(105)$ & 0.149 \\
\hline $\begin{array}{l}\text { Annual household income: } \\
\text { less than US\$25000, \% (n) }\end{array}$ & $24.5(204)$ & $21.2(151)$ & $43.8(53)$ & $<0.001$ \\
\hline $\begin{array}{l}\text { Difficulty paying monthly bills: } \\
\text { somewhat or very difficult, \% (n) }\end{array}$ & $31.7(265)$ & $28.5(203)$ & $51.3(62)$ & $<0.001$ \\
\hline \multicolumn{5}{|l|}{ Health history } \\
\hline Primary diagnosis at index hospitalisation & & & & $<0.001$ \\
\hline ACS, \% (n) & $64.9(541)$ & $69.7(497)$ & $36.4(44)$ & \\
\hline ADHF, \% (n) & $28.4(237)$ & $24.8(177)$ & $49.6(60)$ & \\
\hline Both, \% (n) & $6.7(56)$ & $5.5(39)$ & $14.0(17)$ & \\
\hline $\begin{array}{l}\text { Comorbidity (Elixhauser Index), median } \\
\text { (Q1-Q3) }\end{array}$ & $12.0(5-20)$ & $11.0(4-18)$ & $20.0(12-25)$ & $<0.001$ \\
\hline $\begin{array}{l}\text { Depressive symptoms (PHQ-8) } \\
\text { (possible range 0-24), \% (n) }\end{array}$ & & & & $<0.001$ \\
\hline Severe cognitive impairment (8-10) & 0 & 0 & 0 & \\
\hline $\begin{array}{l}\text { Outpatient visits (past } 12 \text { months), median } \\
\text { (Q1-Q3) }\end{array}$ & $6.0(4-12)$ & $6.0(4-12)$ & $7.0(4-12)$ & 0.050 \\
\hline ED visits (past 12 months), median (Q1-Q3) & $0.0(0-1)$ & $0.0(0-1)$ & $1.0(0-2)$ & $<0.001$ \\
\hline $\begin{array}{l}\text { Hospitalisations (past } 12 \text { months), median } \\
\text { (Q1-Q3) }\end{array}$ & $0.0(0-2)$ & $0.0(0-1)$ & $1.0(0-3)$ & $<0.001$ \\
\hline $\begin{array}{l}\text { Length of hospital stay (days), median (Q1- } \\
\text { Q3) }\end{array}$ & $3.0(2-5)$ & $3.0(2-5)$ & $6.0(4-9)$ & $<0.001$ \\
\hline
\end{tabular}

BHLS, Brief Health Literacy Screen; ED, emergency department; ESSI, ENRICHD Social Support Inventory; HHC, home healthcare; PHQ-8, Patient Health Questionnaire-8; SPMSQ, Short Portable Mental Status Questionnaire.

differential patterns using the vulnerability categories in the propensity-matched subsample.

\section{Patient characteristics associated with changes in vulnerability}

Regardless of the time periods, preceding vulnerability (at baseline or 30 days postdischarge) and HHC referral accounted for $14 \%-16 \%$ of the variance in subsequent change in vulnerability $(\mathrm{p}<0.001)$, while patient variables accounted for an additional $6 \%$ of this variance $(\mathrm{p}<0.001)$. During each time period, older patients (beta $=0.12-0.14$, $\mathrm{p}<0.001)$ and patients with more outpatient visits in the past 12 months (beta $=0.08-0.10, \mathrm{p}<0.05$ ) had a greater increase in vulnerability. Patients with more hospitalisations in the past 12 months had a greater increase in vulnerability from baseline to 30 days postdischarge (beta $=0.09$, $\mathrm{p}<0.05)$. From 30 days to 90 days postdischarge, patients 
Table 2 Vulnerability percentages by group and assessment time points

\begin{tabular}{|c|c|c|c|c|c|c|c|}
\hline \multirow{2}{*}{$\begin{array}{l}\text { HHC referral } \\
\text { group }\end{array}$} & \multirow{2}{*}{$\begin{array}{l}\text { Vulnerability categories } \\
\text { (VES-13 score) }\end{array}$} & \multicolumn{2}{|l|}{ Baseline } & \multicolumn{2}{|c|}{30 Days postdischarge } & \multicolumn{2}{|c|}{90 Days postdischarge } \\
\hline & & $\%(n)$ & Total N & $\%(n)$ & Total N & $\%(n)$ & Total N \\
\hline \multirow[t]{3}{*}{ Overall sample } & Not vulnerable (0-2) & $55.9(466)$ & 834 & $60.8(491)$ & 807 & $65.6(514)$ & 784 \\
\hline & Vulnerable (3-6) & $24.9(208)$ & & $20.6(166)$ & & $18.4(144)$ & \\
\hline & Extremely vulnerable $(7-10)$ & $19.2(160)$ & & $18.6(150)$ & & $16.1(126)$ & \\
\hline \multirow{3}{*}{$\begin{array}{l}\text { Non-HHC } \\
\text { referred }\end{array}$} & Not vulnerable (0-2) & $59.7(426)$ & 713 & $65.8(455)$ & 692 & $69.2(466)$ & 673 \\
\hline & Vulnerable (3-6) & $24.3(173)$ & & $20.1(139)$ & & $17.4(117)$ & \\
\hline & Extremely vulnerable $(7-10)$ & $16.0(114)$ & & $14.2(98)$ & & $13.4(90)$ & \\
\hline \multirow[t]{3}{*}{$\mathrm{HHC}$ referred } & Not vulnerable (0-2) & $33.1(40)$ & 121 & $31.3(36)$ & 115 & $43.2(48)$ & 111 \\
\hline & Vulnerable (3-6) & $28.9(35)$ & & $23.5(27)$ & & $24.3(27)$ & \\
\hline & Extremely vulnerable (7-10) & $38.0(46)$ & & $45.2(52)$ & & $32.4(36)$ & \\
\hline
\end{tabular}

HHC, home healthcare; VES-13, Vulnerable Elders Survey 13.

with depressive symptoms (beta $=0.11, \mathrm{p}<0.01$ ) and those who were African-American (vs Caucasians) had a greater increase in vulnerability (beta $=0.08, \mathrm{p}<0.05$ ). Table 4 presents these results in details.

\section{DISCUSSION}

To our knowledge, this is the first prospective study that examined postdischarge changes in vulnerability to functional decline among older hospitalised patients with cardiovascular diseases (ACS and/or ADHF), and compared postdischarge vulnerability changes between patients in different postacute care options (self-care versus being referred to HHC). This study has two principal findings. First, dynamic changes in vulnerability occurred after hospital discharge, including an initial deterioration in the first 30 days followed by a gradual improvement from 30 to 90 days. Second, HHC seemed to have a positive effect on facilitating postdischarge improvement in vulnerability in older hospitalised patients from 30 days to 90 days after hospital discharge. In the first 30 days after hospital discharge, after adjusting for baseline vulnerability and patient covariates, HHC-referred patients had more increase (ie, worsening) in vulnerability than non-HHC-referred patients.

Overall, older postdischarge patients with cardiovascular disease showed higher levels of baseline vulnerability $(44.4 \%)$ relative to community-dwelling older adults $(32 \%){ }^{8}$ Vulnerability was particularly prevalent among HHC-referred patients $(66.9 \%)$, which indicates that HHC referral was appropriately made for those with worse functional status. This is possibly related to the similarity between the VES-13 and the assessment used to determine HHC appropriateness, as both focus on functional capacity in ADLs. ${ }^{731}$

Among patients who were referred to HHC, vulnerability first worsened in the first 30 days after hospital

\begin{tabular}{|c|c|c|c|c|c|}
\hline Time period & Sample size & B & $95 \% \mathrm{Cl}$ & Beta & $P$ value \\
\hline \multicolumn{6}{|c|}{ Baseline to 30 days } \\
\hline Full model & 807 & -1.01 & -1.44 to -0.58 & -0.16 & $<0.001$ \\
\hline Propensity & 807 & -1.13 & -1.62 to -0.64 & -0.18 & $<0.001$ \\
\hline Matched & 190 & -1.34 & -2.07 to -0.61 & -0.26 & $<0.001$ \\
\hline \multicolumn{6}{|l|}{ 30-90 days } \\
\hline Full model & 757 & +0.40 & +0.80 to +0.01 & +0.07 & 0.055 \\
\hline Propensity & 757 & +0.62 & +0.17 to +1.07 & +0.11 & 0.007 \\
\hline Matched & 168 & +0.83 & +0.20 to +1.45 & +0.19 & 0.010 \\
\hline \multicolumn{6}{|c|}{ Baseline to 90 days } \\
\hline Full model & 784 & -0.30 & -0.75 to +0.14 & -0.05 & 0.185 \\
\hline Propensity & 784 & -0.33 & -0.84 to +0.17 & -0.05 & 0.197 \\
\hline Matched & 178 & -0.29 & -0.99 to +0.41 & -0.06 & 0.409 \\
\hline
\end{tabular}

$\mathrm{B}$ are raw regression weights; beta' are standardised regression weights.

VES-13, Vulnerable Elders Survey 13. 


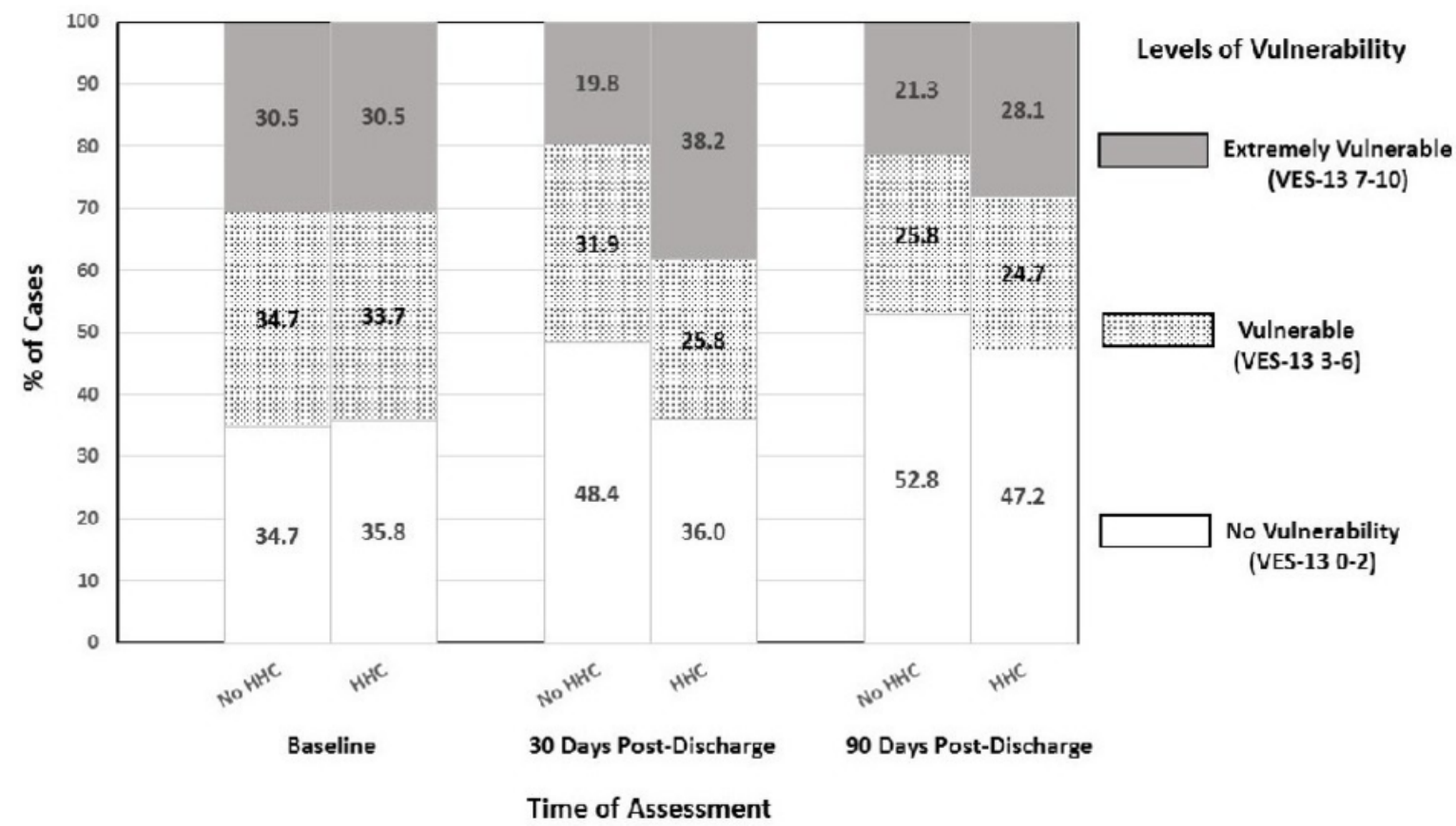

Figure 2 Vulnerability categories at each time of assessment for a group referred to home healthcare propensity matched with a group not referred to home healthcare (n=95 per group). VES-13, Vulnerable Elders Survey 13.

discharge then gradually improved in the following 60 days, suggesting three interesting points.

One is the dynamic nature of physical function related to vulnerability and physical frailty-a phenotype focused on objective physiological changes that is closely intertwined with vulnerability. ${ }^{11}{ }^{32-37}$ As shown in the groundbreaking study by Gill et al, ${ }^{11}$ community-dwelling older adults experienced frequent transitions in frailty over a period of 4.5 years. Similar findings on transitions and changes in vulnerability and physical frailty were also reported in several longitudinal cohort studies with community-dwelling older adults, ${ }^{32-34} 38$ indicating potential for targeted interventions.

Second, despite the recent hospitalisation, older patients with cardiovascular disease still improved in vulnerability to a degree that was lower (ie, better) than their prehospitalisation baseline. In natural conditions without interventions, community-dwelling older adults are more likely to increase (rather than decrease) in their functional decline. ${ }^{39}$ As such, the high prevalence of baseline vulnerability among HHC-referred patients $(67 \%)$ indicates that their natural trajectory of postdischarge vulnerability change would be more likely to be worsening than improving, if no interventions had been provided. The absolute changes in vulnerability (table 2) indicated that all patients improved in vulnerability. This finding challenged the traditional view that little can be done to facilitate functional improvement in vulnerable older patients. Although older adults are often discharged from the hospital with worse functional status than their prehospitalisation baseline,$^{40}$ there is still room for functional improvement with targeted and intensive postacute services. Baseline vulnerability and physical frailty can be used to identify patients who are likely to respond (or not) to certain postacute services.

Third, the comparison between HHC-referred and non-HHC-referred patients (tables 3 and 4) revealed that, in the initial 30 days after hospital discharge, HHC-referred subjects had substantially more worsening in vulnerability than the non-HHC-referred group (VES-13 score change: $\mathrm{B}=-1.34$ (-0.61, -2.07); total 10 points), after controlling for baseline vulnerability and potential covariates. This difference in increased vulnerability could translate to a $37 \%$ higher likelihood of 5 -year functional decline ${ }^{13}$ and a $53 \%$ higher likelihood of in-hospital complications or death. ${ }^{10}{ }^{41}$ In fact, vulnerability worsening in the first 30 days after hospital discharge may be the reason why HHC-referred patients with heart failure had higher rates of 30-day readmission and mortality after hospital discharge compared with their propensity scorematched non-HHC-referred counterparts. ${ }^{42}$

This result is intriguing, because at face value, it seems that HHC is counterproductive for older hospitalised patients in the initial 30 days after discharge. However, the impact of HHC on postdischarge vulnerability change may be related to the timing and visit intensity of HHC services provided for each patient. Recent evidence has shown that postacute HHC, when provided within the first week after discharge, reduces the hazard for 30-day hospital readmission by $39 \% .{ }^{43}$ This means that, for older hospitalised patients, timely provision of supportive care in the immediate postdischarge period is key to overall postacute functional improvement.

The intensity of HHC is also critical to its effect on vulnerability and outcomes such as rehospitalisation. Medicare patients who received at least 22 days of HHC 
Table 4 Association of patient characteristics with changes in vulnerability after controlling for initial VES-13 scores and $\mathrm{HHC}$ referral in linear regression

\begin{tabular}{|c|c|c|c|}
\hline \multirow[b]{2}{*}{ Characteristics } & \multicolumn{3}{|c|}{ Change period } \\
\hline & $\begin{array}{l}\text { Baseline to } \\
30 \text { days }\end{array}$ & $\begin{array}{l}30- \\
90 \text { days }\end{array}$ & $\begin{array}{l}\text { Baseline } \\
\text { to } 90 \text { days }\end{array}$ \\
\hline VES-13 score (baseline) & $-0.54 \dagger$ & & $-0.51 \dagger$ \\
\hline VES-13 score (30 days) & & $-0.50 \dagger$ & \\
\hline HHC referral & $0.16+$ & $-0.07^{*}$ & 0.05 \\
\hline \multicolumn{4}{|l|}{ Hospital admission variables } \\
\hline Age & $0.14 \dagger$ & $0.12 \dagger$ & $0.14 \dagger$ \\
\hline Female & 0.05 & 0.03 & 0.04 \\
\hline Health literacy (BHLS score) & $<0.01$ & -0.02 & $<0.01$ \\
\hline Year of education & 0.06 & 0.01 & 0.04 \\
\hline Difficulty paying bills & 0.03 & 0.01 & 0.03 \\
\hline Married/living with partner & 0.01 & -0.02 & -0.03 \\
\hline Race: African-American & -0.03 & $0.08 \ddagger$ & 0.05 \\
\hline Race: Other & -0.04 & -0.04 & -0.04 \\
\hline Annual household income & -0.08 & -0.03 & -0.05 \\
\hline Social support (ESSI score) & -0.05 & $<0.01$ & -0.04 \\
\hline $\begin{array}{l}\text { Depressive symptoms (PHQ } \\
\text { score) }\end{array}$ & 0.04 & $0.11 \S$ & 0.02 \\
\hline $\begin{array}{l}\text { Cognitive functioning (SPMSQ } \\
\text { score) }\end{array}$ & -0.04 & 0.04 & 0.01 \\
\hline Length of hospital stay & -0.03 & -0.03 & -0.06 \\
\hline Comorbidity (Elixhauser Index) & 0.06 & 0.06 & 0.04 \\
\hline Outpatient visits (past 12 months) & $0.10 \S$ & $0.08 \ddagger$ & $0.09 \ddagger$ \\
\hline Hospitalisations (past 12 months) & $0.09 \ddagger$ & 0.03 & 0.07 \\
\hline Admitting diagnosis: ADHF & -0.02 & 0.03 & $<0.01$ \\
\hline Admitting diagnosis: ACS/ADHF & 0.02 & $<0.01$ & 0.01 \\
\hline
\end{tabular}

Change 30 days from baseline: (baseline VES-13, HHC referral) adjusted $R^{2}=0.15, p<0.001$; (patient factors) $R^{2}$ change $=0.06, p<0.001$; final model: $R^{2}=0.46$, adjusted $R^{2}=0.19, p<0.001$. Change 90 days from 90 days: (30-day VES-13, HHC referral) adjusted $R^{2}=0.13$, $p<0.001$; (patient factors) $R^{2}$ change $=0.06, p<0.001$; final model: $R^{2}=0.43$, adjusted $R^{2}=0.17, p<0.001$. Change 90 days from baseline: (baseline VES-13, HHC referral) adjusted $R^{2}=0.14, p<0.001$ (patient factors) $R^{2}$ change $=0.06, p<0.001$; final model: $R^{2}=0.44$, adjusted $R^{2}=0.18, p<0.001$.

${ }^{*} \mathrm{P}=0.059$.

$\dagger P<0.001$

$\ddagger \mathrm{P}<0.05$.

$\S \mathrm{P}<0.01$

ACS, acute coronary syndrome; ADHF, acute decompensated heart failure; BHLS, Brief Health Literacy Screen; ESSI, ENRICHD Social Support Inventory; HHC, home healthcare; PHQ, Patient Health Questionnaire; SPMSQ, Short Portable Mental Status Questionnaire; VES-13, Vulnerable Elders Surveys 13.

or four skilled nursing visits were less $(13 \%)$ likely to be rehospitalised at 90 days after discharge from HHC. ${ }^{20}$ In addition, patients who received at least 2 months of HHC spent 8 months longer at home before nursing home placement, compared with those who received no or shorter duration of HHC. ${ }^{44}$ On the contrary, patients who did not receive enough HHC (as deemed by family members) were 1.8 times more likely to die. ${ }^{21}$ Since the current study did not include measures of the timing (eg, when HHC services were provided) or visit intensity of
HHC (eg, how many home visits of each involved discipline in HHC were provided in real time after discharge), it is unknown if the delayed improvement in vulnerability was due to (1) late or inadequate HHC provided in the first week (or 30 days) after hospital discharge or (2) null effect of HHC on vulnerability changes in this period even with early and intensive HHC.

The effects of home-based care on improving functional decline and reducing unnecessary healthcare utilisation have been noted in multiple studies. ${ }^{22} 4546$ However, these studies were conducted in different countries, where substantial differences exist in the eligibility for and delivery models of HHC. ${ }^{22}{ }^{45}{ }^{46}$ For example, in the USA, one has to be verified as homebound by a physician to be eligible for $\mathrm{HHC},{ }^{25}$ and $\mathrm{HHC}$ is often provided by for-profit agencies $(80 \%) .{ }^{16}$ In countries with universal health insurance such as the UK, Denmark and Australia, preventive home-based services are included in the national health policy for all older adults with needs, regardless of homebound status. ${ }^{47}$ Furthermore, HHC in the USA is primarily used as a short-term postacute care service. ${ }^{14} 48$ On average, a US patient receives $34 \mathrm{HHC}$ visits per episode,${ }^{1415}$ when evidence has shown that at least 40 home visits are needed to prevent adverse events, such as a nursing home admission. ${ }^{46}$ This suggests that participants in this study may not have not received enough postacute HHC in the first 30 days after hospital discharge to impact their vulnerability status, leading to a delayed recovery in vulnerability. However, the intensity of HHC services varies by person and the effects of HHC on any patient outcome would need to be examined in the context of type and length of services provided.

Lastly, findings in this study support the importance of baseline status to longitudinal changes in vulnerability. Among community-dwelling older adults and recently injured older patients, baseline level of vulnerability or physical frailty is the predominant predictor of subsequent changes in physical function, ADL disability and survival in the following 18 to 54 months. ${ }^{11}{ }^{450}$ Thus, interventions for vulnerable older adults should also focus on maintaining current functional level and avoiding stressors (eg, illness exacerbations and hospitalisations), as each episode of illness and hospitalisation was associated with functional decline and loss of independence. ${ }^{405152}$ Older patients with a higher frequency of healthcare utilisation in this study were more likely to experience an increase in their vulnerability after hospital discharge, which, in turn, increases the need for health services. This highlights the burden of vulnerability and chronic cardiac conditions on increasing health service use.

\section{Limitations and directions for future research}

This study was not originally designed to compare differences in postdischarge vulnerability changes among patients in different postacute care settings. However, given the paucity of data on postdischarge changes in vulnerability among older patients in different postacute care settings, findings in the current study should still be 
valuable but need to be interpreted with consideration of the following limitations.

First, data on the timing and intensity of specific HHC services (eg, skilled nursing, physical/occupational therapy and home health aides) were not available. Such information is critical to future development of precise HHC interventions aimed at facilitating postdischarge functional recovery. For example, some patients may have only received a few visits to check vital signs, while others may have received intensive physical therapy. As noted in a report that calculated the total number of days enrolled in HHC during $2007,{ }^{53}$ the mean of accumulated HHC service per patient per year in the USA is 315 days ( $\mathrm{SD}=33.1)$ with a median of 70 days, indicating large variation in HHC delivery. Because the variation in HHC services is likely to influence the effect of HHC on vulnerability change, future studies should employ the randomised controlled design and include specific measures of HHC services (ie, timing, frequency/intensity and type of services). Second, we focused on postdischarge vulnerability changes for 90 days after hospital discharge, yet recovery in vulnerability and physical function can last for years. ${ }^{54}$ Future research should examine changes in vulnerability with frequent measures across a longer follow-up period. Third, because the VES-13 is a self-report tool, some participants may underestimate their vulnerability due to inherent fears of nursing home placement or other self-report bias, especially when asked to consider their abilities prior to hospitalisation (baseline measure). Future studies should incorporate objective, performance-based measures of vulnerability and frailty (eg, gait speed, hand-grip strength) to augment self-report measures. ${ }^{55}$ Fourth, patients with visual, hearing and significant cognitive impairment and patients without follow-up data on vulnerability were not included in this study, which may have introduced selection bias and limits the generalisability of findings. However, sample characteristics (ie, age, diagnosis, race, education, marital status, difficulty paying bills, cognitive function and depressive symptoms) and baseline level of vulnerability of sample in this study (excluding patients without follow-up data on vulnerability) were comparable with those of the sample in the original study, ${ }^{23}$ other than a lower proportion of female ( $40 \%$ vs $47 \%$ ). Lastly, we used propensity score matching to control for observable confounding;however, there might be unmeasured confounding and residual bias from measured confounders that was not controlled for.

\section{CONCLUSION}

Nearly half of older hospitalised patients with cardiovascular disease were vulnerable at prehospitalisation baseline. Patients discharged home with an HHC referral, despite being more vulnerable at prehospitalisation baseline and having delayed recovery in vulnerability in the initial 30 days after discharge, improved substantially from 30 to 90 days after hospital discharge. At 90 days after hospital discharge, all patients improved in vulnerability to a degree that was lower (ie, better) than the prehospitalisation baseline. Future research should examine how the pattern, frequency and intensity of HHC services affect postdischarge vulnerability improvement in older patients with cardiovascular disease. While more research is needed, this finding suggests that HHC may facilitate postdischarge improvement in vulnerability in older patients with cardiovascular disease from 30 to 90 days after hospital discharge.

\section{Author affiliations}

${ }^{1}$ University of Rochester Medical Center, School of Nursing, Rochester, New York, USA

${ }^{2}$ School of Nursing, Vanderbilt University, Nashville, Tennessee, USA

${ }^{3}$ Department of Biostatistics, Vanderbilt University Medical Center, Nashville, Tennessee, USA

${ }^{4}$ Department of Psychiatry, Vanderbilt University Medical Center, Nashville, Tennessee, USA

${ }^{5}$ Department of Medicine, Vanderbilt University Medical Center, Nashville, Tennessee, USA

${ }^{6}$ Center for Quality Aging, Vanderbilt University Medical Center, Nashville, Tennessee, USA

${ }^{7}$ Geriatric Research, Education and Clinical Center (GRECC), VA Tennessee Valley Healthcare System, Nashville, Tennessee, USA

${ }^{8}$ Center for Clinical Quality and Implementation Research, Vanderbilt University Medical Center, Nashville, Tennessee, USA

Acknowledgements We thank all the participants in the original prospective study for their contribution.

Contributors JW was responsible for the design, analysis, drafting and revision of this manuscript. MSD was responsible for the analysis and revision of this manuscript. SPB, CAM, SFS, SK was responsible for the design and revision of this manuscript.

Funding This work was supported by the National Institute of Health, National Heart, Lung, and Blood Institute (R01 HL109388-06) to SK; National Institute on Aging (K23 AG048347-03) to SPB; the Vanderbilt University School of Nursing PostDoctoral Fund to JW, and in part by grant 2 UL1 TR000445-06 from the National Center for Advancing Translational Sciences.

Disclaimer The content is solely the responsibility of the authors and does not necessarily represent the official views of the National Institutes of Health. This manuscript has not been presented prior to this submission.

Competing interests None declared.

Patient consent for publication Not required.

Ethics approval Vanderbilt University Institutional Review Board (VU IRB).

Provenance and peer review Not commissioned; externally peer reviewed.

Data sharing statement There is no unpublished data-for example, technical appendix, statistical code and dataset—available that are relevant to this specific secondary analysis study.

Open access This is an open access article distributed in accordance with the Creative Commons Attribution Non Commercial (CC BY-NC 4.0) license, which permits others to distribute, remix, adapt, build upon this work non-commercially, and license their derivative works on different terms, provided the original work is properly cited, appropriate credit is given, any changes made indicated, and the use is non-commercial. See: http://creativecommons.org/licenses/by-nc/4.0/.

\section{REFERENCES}

1. Administration on Aging Administration. A Profile of Older Americans: 2014. 2014 http://www.aoa.acl.gov/Aging_Statistics/Profile/2014/ docs/2014-Profile.pdf (Retried 1 Aug 2017).

2. Weiss AJ (Truven Health Analytics), Elixhauser A (AHRQ). Overview of Hospital Stays in the United States, 2012. HCUP Statistical Brief \#180. Rockville, MD: Agency for Healthcare Research and Quality, 2014. http://www.hcup-us.ahrq.gov/reports/statbriefs/sb180Hospitalizations-United-States2012.pdf. (Retried 1 Aug 2017).

3. Bui AL, Horwich TB, Fonarow GC. Epidemiology and risk profile of heart failure. Nat Rev Cardiol 2011;8:30-41.

4. Kripalani S, Jackson AT, Schnipper JL, et al. Promoting effective transitions of care at hospital discharge: a review of key issues for hospitalists. J Hosp Med 2007;2:314-23. 
5. Gheorghiade M, Pang PS. Acute heart failure syndromes. J Am Coll Cardiol 2009;53:557-73.

6. Ross JS, Mulvey GK, Stauffer B, et al. Statistical models and patient predictors of readmission for heart failure: a systematic review. Arch Intern Med 2008;168:1371-86.

7. Saliba D, Elliott M, Rubenstein LZ, et al. The Vulnerable Elders Survey: a tool for identifying vulnerable older people in the community. J Am Geriatr Soc 2001;49:1691-9.

8. Bell SP, Schnelle J, Nwosu SK, et al. Development of a multivariable model to predict vulnerability in older American patients hospitalised with cardiovascular disease. BMJ Open 2015;5:e008122.

9. Luciani A, Ascione G, Bertuzzi C, et al. Detecting disabilities in older patients with cancer: comparison between comprehensive geriatric assessment and vulnerable elders survey-13. J Clin Oncol 2010;28:2046-50.

10. Min L, Ubhayakar N, Saliba D, et al. The Vulnerable Elders Survey-13 predicts hospital complications and mortality in older adults with traumatic injury: a pilot study. J Am Geriatr Soc 2011;59:1471-6.

11. Gill TM, Gahbauer EA, Allore HG, et al. Transitions between frailty states among community-living older persons. Arch Intern Med 2006;166:418-23.

12. Beddoes-Ley $L$, Khaw D, Duke M, et al. A profile of four patterns of vulnerability to functional decline in older general medicine patients in Victoria, Australia: a cross sectional survey. BMC Geriatr 2016;16:150.

13. Min L, Yoon W, Mariano J, et al. The vulnerable elders-13 survey predicts 5-year functional decline and mortality outcomes in older ambulatory care patients. J Am Geriatr Soc 2009;57:2070-6.

14. Medicare Payment Advisory Commission. Report to the Congress, Medicare Payment Policy: Medicare Payment Advisory Commission, 2017.

15. Medicare Payment Advisory Commission. A data book: healthcare spending and the Medicare program. Washington (DC): MedPAC, 2016.

16. Harris-Kojetin L, Sengupta M, Park-Lee E, et al. Long-term care providers and services users in the united states: Data from the national study of long-term care providers, 2013-2014. National center for health statistics. Vital Health Stat 3(38) 2016:105.

17. Centers for Medicare \& Medicaid Services (CMS). Home health care: what it is and what to expect. $2015 \mathrm{http}: / / \mathrm{www}$.medicare.gov/whatmedicare-covers/home-health-care/home-health-care-what-is-itwhat-to-expect.html (Accessed 1 Aug 2017).

18. Madigan EA, Gordon N, Fortinsky RH, et al. Predictors of functional capacity changes in a US population of Medicare home health care $(\mathrm{HHC})$ patients with heart failure (HF). Arch Gerontol Geriatr 2012;54:e300-e306.

19. O'Connor M, Hanlon A, Naylor MD, et al. The impact of home health length of stay and number of skilled nursing visits on hospitalization among Medicare-reimbursed skilled home health beneficiaries. Res Nurs Health 2015;38:257-67.

20. Nanda A, Bourbonniere M, Wetle T, et al. Home care in the last year of life: family member perceptions of unmet need associated with last place of care. J Am Med Dir Assoc 2010;11:21-5.

21. Xiao R, Miller JA, Zafirau WJ, et al. Impact of Home Health Care on Health Care Resource Utilization Following Hospital Discharge: A Cohort Study. Am J Med 2018;131.

22. Lohman MC, Scherer EA, Whiteman KL, et al. Factors Associated With Accelerated Hospitalization and Re-hospitalization Among Medicare Home Health Patients. J Gerontol A Biol Sci Med Sci 2018;73: glw335-glw35.

23. Mohile SG, Bylow K, Dale W, et al. A pilot study of the vulnerable elders survey-13 compared with the comprehensive geriatric assessment for identifying disability in older patients with prostate cancer who receive androgen ablation. Cancer 2007;109:802-10.

24. Meyers AG, Salanitro A, Wallston KA, et al. Determinants of health after hospital discharge: rationale and design of the Vanderbilt Inpatient Cohort Study (VICS). BMC Health Serv Res 2014;14:10.

25. Centers for Medicare and Medicaid Services (CMS). Certifying Patients for the Medicare Home Health Benefit, Department of Health and Human Services. 2013 https://www.cms.gov/Outreach-andEducation/Medicare-Learning-Network-MLN/MLNMattersArticles/ Downloads/SE1436.pdf (Retried 19 Oct 2017).

26. Chew LD, Bradley KA, Boyko EJ. Brief questions to identify patients with inadequate health literacy. Fam Med 2004;36:588-94.

27. Mitchell PH, Powell L, Blumenthal J, et al. A short social support measure for patients recovering from myocardial infarction: the ENRICHD Social Support Inventory. J Cardiopulm Rehabil 2003;23:398-403.

28. van Walraven $C$, Austin PC, Jennings $A$, et al. A modification of the Elixhauser comorbidity measures into a point system for hospital death using administrative data. Med Care 2009;47:626-33.
29. Kroenke K, Strine TW, Spitzer RL, et al. The PHQ-8 as a measure of current depression in the general population. $J$ Affect Disord 2009;114:163-73.

30. Pfeiffer E. A short portable mental status questionnaire for the assessment of organic brain deficit in elderly patients. J Am Geriatr Soc $1975 ; 23: 433-41$

31. Alper E, O'Malley TA, Greenwald J. Auerbach AD, ed. Hospital discharge and readmission. Waltham, MA: UpToDate, 2017.

32. Trevisan C, Veronese N, Maggi S, et al. Factors influencing transitions between frailty states in elderly adults: The progetto veneto anziani longitudinal study. J Am Geriatr Soc 2017;65:179-84.

33. Bentur N, Sternberg SA, Shuldiner J. Frailty transitions in community dwelling older people. Isr Med Assoc J 2016;18:449-53.

34. Espinoza SE, Jung I, Hazuda H. Frailty transitions in the san antonio longitudinal study of aging. J Am Geriatr Soc 2012;60:652-60.

35. Lang PO, Michel JP, Zekry D. Frailty syndrome: a transitional state in a dynamic process. Gerontology 2009;55:539-49.

36. Fried LP, Tangen CM, Walston J, et al. Frailty in older adults: evidence for a phenotype. J Gerontol A Biol Sci Med Sci 2001;56:M146-M157.

37. Fried LP, Ferrucci L, Darer J, et al. Untangling the concepts of disability, frailty, and comorbidity: Implications for improved targeting and care. J Gerontol A Biol Sci Med Sci 2004;59:M255-M263.

38. Johansen KL, Dalrymple LS, Delgado C, et al. Factors associated with frailty and its trajectory among patients on hemodialysis. Clin J Am Soc Nephrol 2017:12:1100-8.

39. Ali TF, Warkentin LM, Gazala S, et al. Self-reported outcomes in individuals aged 65 and older admitted for treatment to an acute care surgical service: a 6-month prospective cohort study. J Am Geriatr Soc 2015;63:2388-94.

40. Covinsky KE, Palmer RM, Fortinsky RH, et al. Loss of independence in activities of daily living in older adults hospitalized with medical illnesses: increased vulnerability with age. J Am Geriatr Soc 2003:51:451-8.

41. McGee HM, O'Hanlon A, Barker M, et al. Vulnerable older people in the community: relationship between the Vulnerable Elders Survey and health service use. J Am Geriatr Soc 2008;56:8-15.

42. Arundel $\mathrm{C}$, Sheriff $\mathrm{H}$, Bearden DM, et al. Discharge home health services referral and 30-day all-cause readmission in older adults with heart failure. Arch Med Sci 2018;14:995-1002.

43. Carnahan JL, Slaven JE, Callahan CM, et al. Transitions from skilled nursing facility to home: The relationship of early outpatient care to hospital readmission. J Am Med Dir Assoc 2017;18:853-9.

44. Young $Y$, Kalamaras J, Kelly $\mathrm{L}$, et al. Is aging in place delaying nursing home admission? J Am Med Dir Assoc 2015;16:900.e1-900. e6.

45. Ritchie C, Andersen R, Eng J, et al. Implementation of an interdisciplinary, team-based complex care support health care model at an academic medical center: Impact on health care utilization and quality of life. PLoS One 2016;11:e0148096.

46. Stuck AE, Egger M, Hammer A, et al. Home visits to prevent nursing home admission and functional decline in elderly people: systematic review and meta-regression analysis. JAMA 2002;287:1022-8.

47. Byles JE. A thorough going over: evidence for health assessments for older persons. Aust N Z J Public Health 2000;24:117-23.

48. Jones AL, Harris-Kojetin L, Valverde R. Characteristics and use of home health care by men and women aged 65 and over. Hyattsville, MD: National Center for Health Statistics, 2012.

49. Maxwell CA, Mion LC, Mukherjee K, et al. Preinjury physical frailty and cognitive impairment among geriatric trauma patients determine postinjury functional recovery and survival. J Trauma Acute Care Surg 2016;80:195-203.

50. Vermeulen J, Neyens JC, van Rossum E, et al. Predicting ADL disability in community-dwelling elderly people using physical frailty indicators: a systematic review. BMC Geriatr 2011;11:33.

51. Gill TM, Allore HG, Holford TR, et al. Hospitalization, restricted activity, and the development of disability among older persons. JAMA 2004;292:2115-24.

52. Covinsky KE, Pierluissi E, Johnston CB. Hospitalization-associated disability: "She was probably able to ambulate, but l'm not sure". JAMA 2011;306:1782-93.

53. Caffrey C. Home health care and discharged hospice care patients: United states, 2000 and 2007: Us department of health and human services, centers for disease control and prevention, national center for health statistics. 2011

54. Boyd CM, Landefeld CS, Counsell SR, et al. Recovery of activities of daily living in older adults after hospitalization for acute medical illness. J Am Geriatr Soc 2008;56:2171-9.

55. Stall N, Nowaczynski M, Sinha SK. Systematic review of outcomes from home-based primary care programs for homebound older adults. J Am Geriatr Soc 2014;62:2243-51. 